\title{
Plasmonic absorption properties of bimetallic metamaterials
}

\author{
Evangelos Atmatzakis, ${ }^{1, *}$ Nikitas Papasimakis, ${ }^{1, \dagger}$ and Nikolay I. Zheludev ${ }^{1,2}$ \\ ${ }^{1}$ Optoelectronics Research Centre and Centre for Photonic Metamaterials, \\ University of Southampton, Southampton SO17 1BJ, United Kingdom \\ ${ }^{2}$ Centre for Disruptive Photonic Technologies, School of Physical and Mathematical Sciences and the Photonics Institute, \\ Nanyang Technological University, Singapore 637371
}

(Dated: December 28, 2016)

\begin{abstract}
We demonstrate polarization controlled resonant absorption in plasmonic bimetallic metamaterials. We fabricate and experimentally characterize $\mathrm{Au} / \mathrm{Ni}$ ring resonator arrays, where by varying the wavelength and polarization of the incident wave, local electromagnetic fields and dissipation can be suppressed or enhanced in the $\mathrm{Au}$ and $\mathrm{Ni}$ areas of the rings.
\end{abstract}

\section{INTRODUCTION}

Plasmonic metamaterials exhibit extraordinary properties that often require a resonant dispersion. However, intrinsic losses in plasmonic systems usually damp the optical response and result in weaker resonances. On the other hand, there are applications which require strong optical absorption and maximizing the dissipation losses is actually desirable [1]. A few examples include the use of plasmonic metamaterials in order to enhance photoemission [2-4], form water vapour [5], generate electricity $[6,7]$ and thermoelectrically driven magnetic pulses [8, 9]. In principle, the increased absorption cross-section that nanostructures offer [10] is an effective mechanism of energy exchange between light and matter. Since absorption depends on geometrical and material properties, realizing a dynamically controlled system can be challenging. For example, it has been demonstrated that a thin-film perfect absorber with tunable absorption can be realized by taking advantage of interference effects between two incident light beams [11, 12]. In this work we follow a different approach and propose the use of a composite resonator, comprising materials with significantly different optical conductivities, which enables the tailoring of the system's absorption by manipulating the optical mode and promoting current flow in either part of the resonator. We introduce a plasmonic metamaterial consisting of bimetallic $\mathrm{Au} / \mathrm{Ni}$ ring resonators, and study its absorption properties. We demonstrate strong plasmonic resonances which depend on the composition of the constituent metals, as well as the wavelength and polarization of the incident wave. Due to the non-uniformity of the unit-cell, the two ring sections exhibit spectrally separated and polarization sensitive optical absorption.

\section{DESIGN AND FABRICATION}

We study arrays of bimetallic ring resonators with $185 \mathrm{~nm}$ mean diameter (65 nm of linewidth) consisting of Au and $\mathrm{Ni}$ (see Fig. 1). The angle of the Ni sector controls the composition of the two metals in the ring. Four samples have been fabricated with $\mathrm{Ni}$ sectors that span over $45^{\circ}, 90^{\circ}, 135^{\circ}$ and $180^{\circ}$. To simplify our study, the metamaterial unit cell was fixed at $400 \mathrm{~nm}$ for all ring compositions, allowing to achieve a high filling factor for the metallic ring 


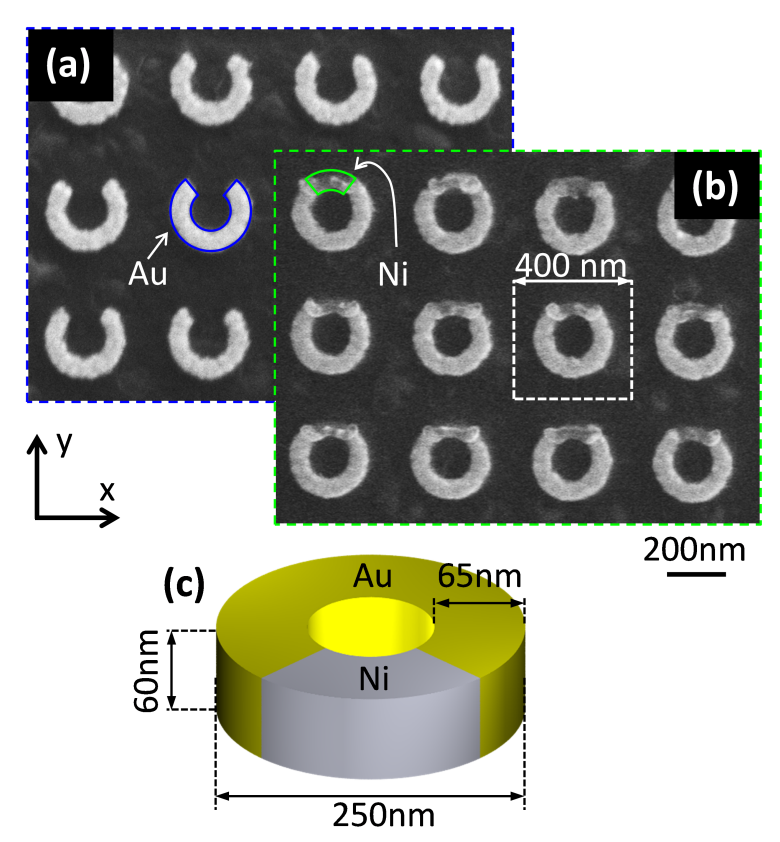

FIG. 1: An example of bimetallic ring resonators consisting of 3/4 Au and 1/4 Ni. (a) SEM image of an array of the Au ring sector and (b) the full bimetallic ring resonator. c Schematic of the ring that has been simulated numerically.

resonators. The samples were fabricated by a two-step electron-beam lithography method on a glass substrate. Thin metal films with thickness of $60 \mathrm{~nm}$ were thermally evaporated on the substrate and lifted-off to reveal each sector of the ring. Problems with the adhesion of gold to the substrate were addressed by avoiding the use of a sonication bath during lift-off. To facilitate a faster lift-off process, a $50 \mathrm{~nm}$ thick layer of co-polymer was deposited prior to $200 \mathrm{~nm}$ of PMMA. Prior to the e-beam writing stage, a thin conductive polymer layer (E-spacer 300Z) was deposited on the PMMA resist and was subsequently dissolved in water before development. As it can be seen in Fig. 1, during the first step Au split-rings are formed (blue outline) and subsequently the Ni part (green outline) is placed to complete the ring. In order to account for fabrication inaccuracies and alignment limitations the two sectors were designed to overlap over a small area around the junctions. A good contact between the metal sectors is essential in order for the system to respond according to design, as any gap caused by fabrication error would strongly affect the position and strength of plasmonic resonances. The surface roughness of the fabricated samples ranges between 5 and 20 nm (RMS) and is present mainly at the nickel sections. As Ni tends to form large grains, small deformations usually develop on its surface, resulting in high values of roughness. However, these deformations are limited only to the direction normal to the metamaterial plane as all other surfaces are strictly defined during the lithography process or by the substrate.

\section{RESULTS}

The fabricated samples were characterized optically using a micro-spectro-photometer system. Transmission and reflection was measured in the wavelength range of $500 \mathrm{~nm}-2000 \mathrm{~nm}$. The samples were illuminated by linearly 

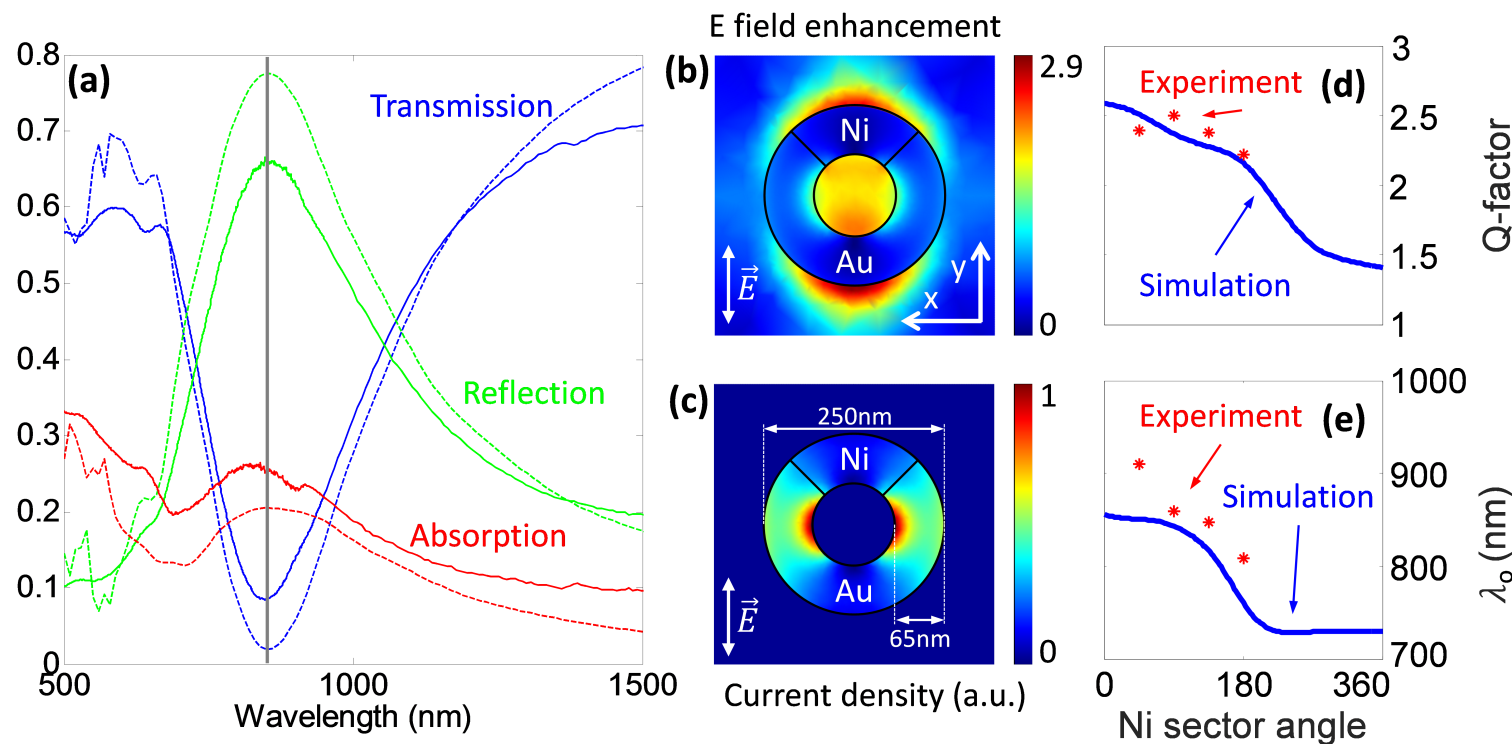

Current density (a.u.)

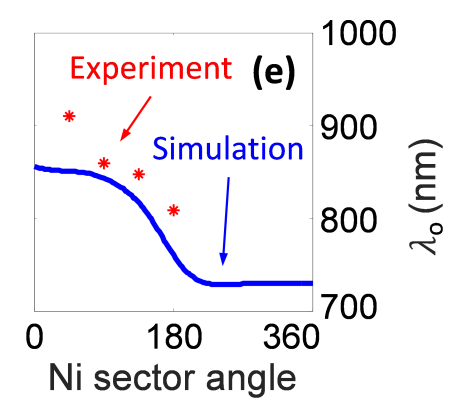

FIG. 2: (a) Transmission, reflection and absorption spectra of a resonator with a $90^{\circ} \mathrm{Ni}$ sector and $270^{\circ}$ Au sector. Solid lines represent fabricated samples and dashed lines the numerical simulations. (b) Electric field enhancement map on the vicinity of ring normalized to the incident field magnitude. (c) Current density distribution along the ring. (d) Numerical (blue) and experimental (red) measurement of Q-factor for 4 different bimetallic resonators where the Ni sectors extend in $45^{\circ}, 90^{\circ}, 135^{\circ}$ and $180^{\circ}$. (e) The spectral position of the corresponding peaks of absorption in simulation and experiment.

polarized white light provided by a halogen lamp. Imaging took place in a scanning electron microscope. Numerical simulations of the metamaterial's plasmonic response were carried out using Comsol Multiphysics, a commercial finiteelement solver. The metamaterial was simulated as an infinite 2D array of the bimetallic ring resonators. Values for the permittivity of $\mathrm{Au}$ and Ni were acquired from [13].

Upon excitation of the ring with light linearly polarized along the axis of symmetry (y-axis in Fig. 1), a strong plasmonic resonance appears centered around $850 \mathrm{~nm}$ (Fig. 2a). This resonance corresponds to the lowest-order dipole mode of the ring as inferred by examining the field maps of Fig. 2b, where two areas of electric field enhancement can be identified across the ring. Accordingly, the current distribution is at a maximum in the region between the two electric field enhancement areas (see Fig. 2c). Numerical simulations and experimental measurements appear to match closely according to Fig. 2a. Any discrepancies can be attributed to fabrication inaccuracies that lead to deviation from the design dimensions and inhomogeneous broadening of the plasmonic resonance. In addition, the finite size of the fabricated samples has not been taken into account in the simulations where an infinite 2D array of resonators was assumed. The composition of the ring resonators strongly affects the response of the system, as the two metals have different optical properties. Nickel's complex permittivity has a higher imaginary part in comparison to Au and thus exhibits increased losses due to Joule heating (see also Supplemetary Information). Hence, increasing the size of the Ni sector leads to damping of the resonance and a decrease of the Q-factor (Fig. 2d). At the same time, the position of the resonance peak blue-shifts due to changes in the effective permittivity of the ring (Fig. 2e). Exploiting the difference in optical conductivities between the two metals that constitute the ring resonator, we can realize a 
system in which the level and spatial distribution of absorption can be dynamically controlled. In such bimetallic resonators, optical absorption is driven by dissipation losses which depend on both the sector size of and the current density in each metal. The latter is prescribed by the plasmonic mode that is excited in the ring and is controlled by the polarization and the wavelength of the incident wave. Although larger Ni sectors lead to weaker resonances,

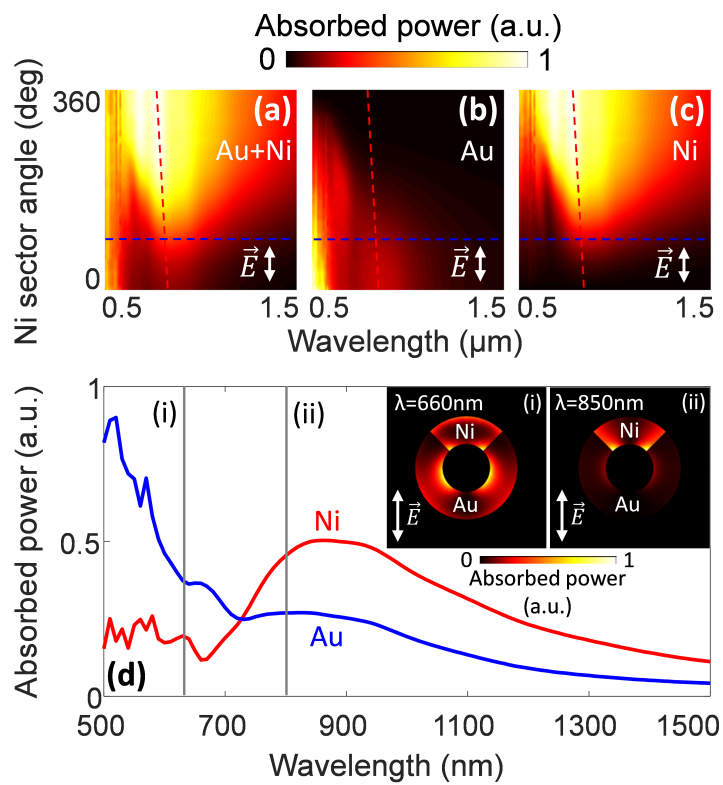

FIG. 3: Spectra of absorption in the full bimetallic ring (a) and the corresponding Au (b) and Ni (c) parts as a function of the angle of the Ni sector. (d) Absorption spectrum of $\mathrm{Au}$ (blue) and $\mathrm{Ni}$ (red) sectors for the case of rings with $90^{\circ} \mathrm{Ni}$ sectors (represented with dashed blue lines in (a),(b) and (c)). The distribution of absorption across the ring is presented as a pair of insets for two characteristic spectral positions, $\lambda_{i}=660 \mathrm{~nm}$ and $\lambda_{i i}=850 \mathrm{~nm}$ marked by the grey lines in (d).

the overall absorption increases (see Fig. 3a) as current is forced to flow through the more "lossy" material. Looking at Figs. 3a-c, we can identify two separate spectral areas of enhanced absorption. At the high frequency end of the spectrum Au appears to absorb more due to higher-order modes that are excited on the ring resonator, but such modes become over-damped for large Ni sectors. On the other hand, the absorption spectrum of the Ni part has its maximum around the resonance wavelength which is represented in Figs. 3a-c by the dashed red line.

Wavelength controlled absorption. As absorption depends on the current density mode that is excited on the resonator, we can engage different absorption regimes by shifting the maxima of the current density towards the more or less lossy regions of the ring. In Fig. 3d, we show that varying the excitation wavelength of a ring with a $90^{\circ} \mathrm{Ni}$ sector enables the shift of absorption from $\mathrm{Au}$ (at shorter wavelengths) to Ni (at the plasmonic resonance). This is further illustrated in the insets to Fig. 3d, where the spatial distribution of absorbed power is presented for two characteristic cases. At $660 \mathrm{~nm}$ absorption occurs mainly in the Au part of the ring, whereas in the Ni region absorption is low. At $850 \mathrm{~nm}$ the situation is reversed: absorption is enhanced in $\mathrm{Ni}$ and suppressed in Au, whereas for both metals losses mainly occur close to the inner circumference of the ring. Finally, the spread of currents away from the Ni sector with decreasing wavelength can be linked to the transitioning from the fundamental mode to higher order ones. 
Polarization controlled absorption. The current configuration of the bimetallic rings at resonance can also be controlled by rotating the polarization azimuth of the incident beam, which allows us to steer the current density towards or away from the lossy Ni section. We illustrate this mechanism of polarization controlled absorption by examining a specific case of a bimetallic ring with a Ni sector of $90^{\circ}$. In Figs. $4 \mathrm{a} \& \mathrm{~b}$, we present the absorption in the $\mathrm{Au}$ and $\mathrm{Ni}$ sectors as a function of the incident wave's polarization. When the excitation beam is linearly polarized and parallel to the y-axis (see Fig. 1), denoted here as $0^{\circ}$, the currents (and hence absorption) in $\mathrm{Ni}$ are at a minimum (Fig. 4c(i)). Rotation of the polarization angle away from this position is accompanied by a shift of the current distribution and the corresponding absorption "hot-spots", engaging larger portions of the Ni section. This leads to an increase of absorption in the Ni sector as seen in Figs. 4a \& b. In contrast, absorption in the Au part does not change significantly due to the large size of the sector that overlaps with the current distribution at any polarization angle. The transitioning from polarization angles between $0^{\circ}$ and $90^{\circ}$ can be seen in Figs. $4 \mathrm{c}(\mathrm{i})$-(iii). The final position, marks the polarization at which the current density and absorption that are induced in the Ni sector are at maximum as seen in Fig. 4b. This method of manipulating the plasmonic mode through the polarization of incident beam enables control not only over the level of absorption but also its spatial distribution.

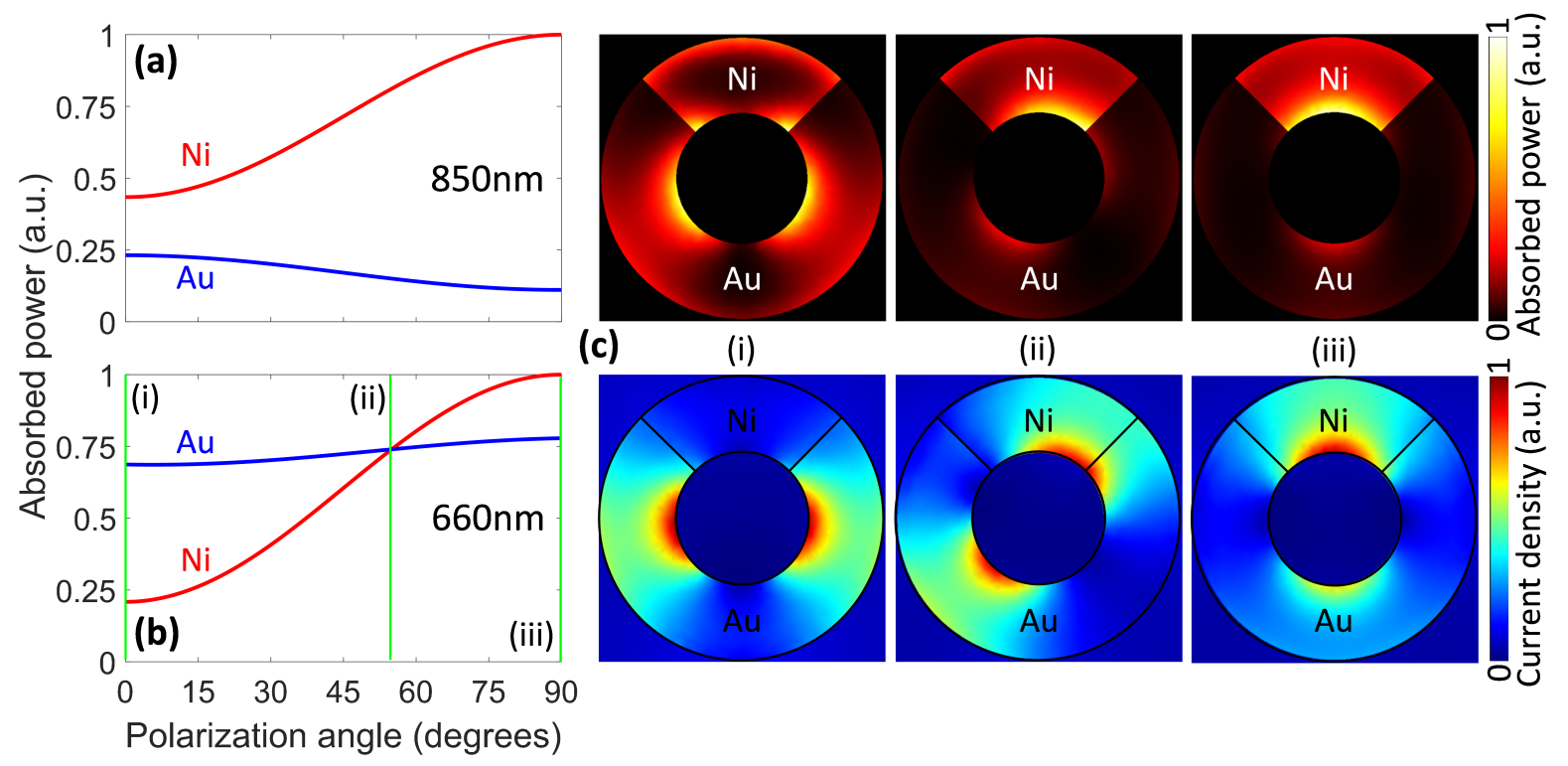

FIG. 4: (a-b) Absorption in Au (blue) and Ni (red) part of a resonator with $90^{\circ} \mathrm{Ni}$ sector at different polarization angles of the incident beam: (a) corresponds to excitation at the resonance of the ring $(850 \mathrm{~nm})$ and (b) at a point of low absorption in $\mathrm{Ni}(660 \mathrm{~nm})$. (c) Distribution of the absorption (upper row) on the ring and the corresponding current density (lower row) for different polarization angles marked by the green lines in (b).

\section{CONCLUSION}

In summary, we have shown that bimetallic ring resonator arrays excited at resonance exhibit strong absorption, which can be controlled by the polarization and wavelength of the incident light. Our results offer an effective way 
to control field enhancement and dissipation losses at prescribed areas of the ring resonators, which can be used in applications where site-specific heating is essential. Finally, bimetallic hybrids exhibit a variety of interesting properties like differential thermal expansion or the ability to generate thermoelectric currents, that can be used, for example, in the generation of $\mathrm{THz}$ magnetic pulses $[8,9]$.

\section{ACKNOWLEDGEMENTS}

The authors would like to thank Anagnostis Tsiatmas and F. Javier Garcia de Abajo for numerous discussions. The authors acknowledge the support of the MOE Singapore (grant MOE2011-T3-1-005), the UKs Engineering and Physical Sciences Research Council (grants EP/G060363/1), and the Leverhulme Trust. The data from this paper can be obtained from the University of Southampton ePrints research repository: http://dx.doi.org/10.5258/SOTON/XXXXXX.

* Electronic address: ea4g11@orc.soton.ac.uk

$\dagger$ Electronic address: n.papasimakis@soton.ac.uk

[1] Mark L. Brongersma, Naomi J. Halas, and Peter Nordlander. Plasmon-induced hot carrier science and technology. Nature Nanotechnology, 10(1):25-34, 2015.

[2] J. Hofmann and W. Steinmann. Plasma resonance in the photoemission of silver. physica status solidi (b), 30(1):K53-K56, 1968.

[3] J. E. Sipe and J. Becher. Surface-plasmon-assisted photoemission. J. Opt. Soc. Am., 71(10):1286-1288, Oct 1981.

[4] S. V. Zhukovsky, V. E. Babicheva, A. V. Uskov, I. E. Protsenko, and A. V. Lavrinenko. Enhanced electron photoemission by collective lattice resonances in plasmonic nanoparticle-array photodetectors and solar cells. Plasmonics, 9(2):283-289, 2013.

[5] O. Neumann, A. S. Urban, J. Day, S. Lal, P. Nordlander, and N. J. Halas. Solar vapor generation enabled by nanoparticles. ACS Nano, 7(1):42-49, 2013.

[6] César Clavero. Plasmon-induced hot-electron generation at nanoparticle/metal-oxide interfaces for photovoltaic and photocatalytic devices. Nature Photonics, 8(2):95-103, 2014.

[7] M. T. Sheldon, J. van de Groep, A. M. Brown, A. Polman, and H. A. Atwater. Plasmoelectric potentials in metal nanostructures. Science, 346(6211):828-832, 2014.

[8] A. Tsiatmas, E. Atmatzakis, N. Papasimakis, V. A. Fedotov, B. Luk'yanchuk, N. I. Zheludev, and F. J. Garca de Abajo. Optical generation of intense ultrashort magnetic pulses at the nanoscale. New Journal of Physics, 15(11):113035, 2013.

[9] G. Vienne, X. Chen, Y. S. Teh, Y. J. Ng, N. O. Chia, and C. P. Ooi. Novel layout of a bi-metallic nanoring for magnetic field pulse generation from light. New Journal of Physics, 17(1):013049, 2015.

[10] C. F. Bohren. How can a particle absorb more than the light incident on it? American Journal of Physics, 51:323-327, 1983.

[11] X. Fang, M. L. Tseng, J.-Y. Ou, K. F. MacDonald, D. P. Tsai, and N. I. Zheludev. Ultrafast all-optical switching via coherent modulation of metamaterial absorption. Applied Physics Letters, 104(14):141102, April 2014.

[12] J. Zhang, K. F. MacDonald, and N. I. Zheludev. Controlling light-with-light without nonlinearity. Light: Science ES Applications, 1(7):e18, 2012. 
[13] E. D. Palik. Handbook of Optical Constants of Solids. Academic Press, San Diego, 1985. 